\title{
PRÁCTICAS SEXUALES EN GESTANTES PERUANAS
}

\author{
Oscar Munares-García1,a, , Flor Alvarado Rodriguez¹,b, Mirian Solís Rojas ${ }^{1, c}$
}

\begin{abstract}
RESUMEN
Objetivo. Identificar las prácticas sexuales durante el embarazo. Métodos. Estudio prospectivo, transversal realizado en 110 gestantes atendidas en el Centro de Salud La Tinguiña, Ica. Se aplicó un cuestionario validado, que evaluaba las prácticas sexuales. Posteriormente se aplicaron estadísticas descriptivas. Resultados. El 69,1\% se encontraba entre los 20 a 34 años. $47,3 \%$ consideró que su vida sexual durante el embarazo fue regular. Al primer trimestre el 100\% indicó tener juegos preliminares la mayoría de las veces, para el segundo trimestre llegó a 59,2\% y para el tercero a 58,9\%. La frecuencia sexual se reduce para los que tenían más de 2 veces por semana, incrementándose a los que tenían cada quince días, de $12,2 \%$ para el segundo trimestre a $39,3 \%$ para el tercer trimestre. Conclusiones. La práctica sexual en el embarazo presenta modificaciones conforme se incrementan los trimestres del embarazo, siendo más marcados para los juegos preliminares y frecuencia sexual.
\end{abstract}

Palabras Clave. Sexualidad; Embarazo; Práctica sexual (Fuente: DeCS BIREME).

\section{SEXUAL PRACTICES IN PERUVIAN GESTANTS}

\begin{abstract}
Objective. Identify sexual practices during pregnancy. Methods. Prospective, cross-sectional study conducted in 110 pregnant women attended at the La Tinguiña Health Center, Ica. A validated questionnaire was applied, which evaluated sexual practices. Subsequently, descriptive statistics were applied. Results. $69,1 \%$ were between 20 and 34 years old. $47,3 \%$ considered that their sexual life during pregnancy was regular. In the first quarter $100 \%$ indicated having preliminary games most of the time, for the second quarter it reached $59,2 \%$ and for the third quarter it reached $58,9 \%$. The sexual frequency is reduced for those who had more times per week, increasing to those who had every fortnight, from $12,2 \%$ for the second quarter to $39,3 \%$ for the third quarter. Conclusions. The sexual practice in pregnancy changes as the quarters increase.
\end{abstract}

Keywords: Sexuality; Pregnancy; Sexual practice (Source: MeSH NLM).

\section{INTRODUCCIÓN}

La actividad sexual es una expresión conductual de la sexualidad personal donde el componente erótico es el más evidente, se caracteriza por el deseo sexual, la práctica sexual y la orientación sexual. La práctica sexual es el conjunto de actividades que las parejas realizan para ejercer su actividad sexual. Sacomori y Cardoso definen la práctica sexual como los diversos tipos de actividades de carácter sexual desde la masturbación, sexo oral, penetración vaginal, penetración anal hasta la estimulación con vibrador, pudiendo ser variadas y de acuerdo a la experiencia ${ }^{1}$.

La práctica sexual sufre cambios durante el embarazo, disminuyendo en su intensidad y frecuencia conforme el embarazo progresa, para luego regularizarse posterior al parto. Rodrigues et $\mathrm{al}^{2}$ sostienen que durante este período, la sexualidad de las embarazadas se ven afectadas por varios factores, como los cambios en la percepción de la imagen corporal, disminución del nivel de energía, presencia de síntomas fisiológicos y molestias corporales, adaptación a las nuevas funciones sociales, calidad de la relación, cambios de humor ${ }^{3}$ entre otros, que pueden ser experimentados por las embarazadas, así como su pareja. Bello et al en un estudio de gestantes, encontraron que la libido aumenta durante el embarazo, pero la frecuencia de orgasmos se reducen ${ }^{4}$. Durante el embarazo se dan cambios en la función sexual que afectan el deseo, la respuesta y el comportamiento sexual, sin embargo para otras el embarazo permite una expresión positiva de la sexualidad $^{5}$. El objetivo de la investigación fue determinar la calidad del sexo antes y durante el embarazo en gestantes de Ica.

\footnotetext{
Departamento Académico de Obstetricia de la Facultad de Medicina de la Universidad Nacional Mayor de San Marcos. Lima-Perú.

a Licenciado en Obstetricia, Doctor en Ciencias de la Salud, Magíster en Salud Pública.

b Licenciada en Obstetricia, Magíster en Obstetricia. Hospital Nacional Daniel Alcides Carrión, Callao, Perú.

c Licenciada en Obstetricia, Magíster en Educación Superior, Doctora en Salud Pública. Docente investigadora Universidad Privada Telesup.

Citar como: Munares-García O, Alvarado F, Solís M. Prácticas sexuales en gestantes peruanas. Rev Peru Investig Matern Perinat 2018; 7(2):41-5.

https://doi.org/10.33421/inmp.2018117
}

Recibido: 07-12-18 Aprobado: 30-12-18 


\section{MATERIAL Y MÉTODOS}

Tipo de estudio. Estudio prospectivo, transversal descriptivo. Área de estudio. Gestantes atendidas en el Centro de Salud La Tinguiña, ubicado en el distrito de La Tinguiña, provincia y distrito de Ica, Perú. Establecimiento perteneciente al Gobierno regional de Ica, atiende a gestantes afiliadas al Seguro Integral de Salud (SIS). Población y muestra. Durante el periodo de estudio se estimó una cantidad de 152 gestantes que acuden al Centro de Salud, de ellos se obtuvo una muestra de 110, que cumplieron con los siguientes criterios de inclusión: aceptaron participar, atendida en el establecimiento, excluyendo analfabetas, gestaciones múltiples y aquellas con algún problema de comprensión del cuestionario, los datos fueron recolectados el mes de agosto de 2012. Variables. Se midieron edad, nivel educativo, estado civil, edad gestacional, así como elementos de la práctica sexual como vida sexual, iniciativa para la práctica sexual, juegos preliminares, frecuencia de actividad sexual, presencia de orgasmo y una calificación final de 0 a 10 sobre su percepción de su vida sexual. Técnicas e instrumentos. Para el estudio se aplicó la técnica del cuestionario estructurado y como instrumento se aplicó un formulario validado por Sacomori ${ }^{3}$. Procedimientos. Inicialmente se identificaron los casos en el establecimiento de salud, se procedió a invitar a las gestantes, luego de determinar los criterios de selección se procedió a firmar el consentimiento informado, posteriormente se aplicó los cuestionarios que medían la práctica sexual, con una duración media de 15 minutos, posterior al procedimientos se brindó consejería sobre práctica sexual posterior al parto. Aspectos éticos. Se salvaguardaron los principios de ética en la investigación, los cuestionarios fueron aplicados de forma anónima, no se registraron nombres, se aplicó consentimiento informado y la base de datos se trabajó con códigos. El proyecto fue aprobado por el Comité de Investigación de la Escuela de Obstetricia de la Universidad Alas Peruanas filial Ica. Análisis de datos. Para las variables cualitativas se empleó distribución de frecuencias y porcentajes, en la edad gestacional se dividieron en tres grupos, el primer trimestre de 1 a 14 semanas, el segundo trimestre de 15 a 28 semanas y el tercer trimestre de 29 a 41 semanas, se aplicaron tablas y figuras para mejor entendimiento.

\section{RESULTADOS}

El $69,1 \%$ se encontraba entre los 20 a 34 años, $40,9 \%$ tenía un nivel educativo secundario, $63,6 \%$ era conviviente y $50,9 \%$ se encontraba en el tercer trimestre del embarazo (Tabla 1).

Con respecto a la vida sexual en general, podemos indicar que un $47,3 \%$ consideró que su vida sexual
Tabla 1. Distribución porcentual de las gestantes, Ica

\begin{tabular}{|c|c|c|}
\hline & $\mathbf{n}$ & $\%$ \\
\hline \multicolumn{3}{|l|}{ Edad (años) } \\
\hline 15 a 19 & 25 & 22,7 \\
\hline 20 a 34 & 76 & 69,1 \\
\hline 35 a 40 & 9 & 8,2 \\
\hline \multicolumn{3}{|l|}{ Nivel educativo } \\
\hline Primaria & 5 & 4,5 \\
\hline Secundaria & 45 & 40,9 \\
\hline Superior Técnica & 21 & 19,1 \\
\hline Superior universitaria & 39 & 35,5 \\
\hline \multicolumn{3}{|l|}{ Estado civil } \\
\hline Soltera & 13 & 11,8 \\
\hline Casada & 27 & 24,5 \\
\hline Conviviente & 70 & 63,6 \\
\hline \multicolumn{3}{|l|}{ Trimestre embarazo } \\
\hline Primer & 5 & 4,5 \\
\hline Segundo & 49 & 44,5 \\
\hline Tercero & 56 & 50,9 \\
\hline Total & 110 & 100,0 \\
\hline
\end{tabular}

durante el embarazo fue regular, y un $32,7 \%$ indicó que fue buena, al evaluarlo por trimestres, se puede apreciar que en el primer trimestre ninguna de las evaluadas indicó que su vida sexual era buena, este evento tiende ser mayor conforme se incrementa los trimestres a ser del $38,8 \%$ para el segundo trimestre a $30,4 \%$ para el tercer trimestre. Con respecto a la iniciativa para la práctica sexual, se puede apreciar que esta fue en general iniciada por ambos $(59,1 \%)$. Con respecto a los juegos preliminares, se puede apreciar que en el $60,9 \%$ fue la mayoría de las veces, este evento que fue total en el primer trimestre, se reduce conforme se incrementan los trimestres llegando al 58,9\% para el tercer trimestre. Con respecto a la frecuencia de la actividad sexual, se puede apreciar que para el primer trimestre es casi similar en todas, incrementándose conforme los trimestres avanzan la práctica mensual, y cada quince días, reduciéndose los de mayor frecuencia como la mayor práctica por semana. Con respecto a la presencia de orgasmo, se puede apreciar que este tiende a ser constante de acuerdo a los trimestres y depende de la posición tomada y se reduce con la penetración profunda (Tabla 2).

Finalmente con respecto a la percepción de la calidad del sexo durante el embarazo, podemos determinar la mediana de la escala se mantiene similar conforme se incrementan los trimestres de gestación, es decir los cambios no son tan marcados (Figura 1). 
Tabla 2. Distribución porcentual de las características de la práctica sexual en el embarazo según trimestres de embarazo

\begin{tabular}{|c|c|c|c|c|}
\hline & Primer & Segundo & Tercer & Total \\
\hline & $n=5$ & $n=49$ & $n=56$ & $n=110$ \\
\hline & $\%$ & $\%$ & $\%$ & $\%$ \\
\hline \multicolumn{5}{|l|}{ Consideración de su vida sexual } \\
\hline Mala & 20,0 & 20,4 & 12,5 & 16,4 \\
\hline Regular & 40,0 & 36,7 & 57,1 & 47,3 \\
\hline Bueno & 0,0 & 38,8 & 30,4 & 32,7 \\
\hline Excelente & 40,0 & 4,1 & 0,0 & 3,6 \\
\hline \multicolumn{5}{|c|}{ Iniciativa para práctica de relaciones sexuales } \\
\hline Los dos & 40,0 & 69,4 & 51,8 & 59,1 \\
\hline Mi pareja & 40,0 & 30,6 & 44,6 & 38,2 \\
\hline Yo misma & 20,0 & 0,0 & 3,6 & 2,7 \\
\hline \multicolumn{5}{|l|}{ Juegos preliminares } \\
\hline Rara vez & 0,0 & 10,2 & 10,7 & 10,0 \\
\hline A veces & 0,0 & 26,5 & 23,2 & 23,6 \\
\hline La mayoría de las veces & 100,0 & 59,2 & 58,9 & 60,9 \\
\hline Siempre & 0,0 & 4,1 & 7,1 & 5,5 \\
\hline \multicolumn{5}{|l|}{ Frecuencia relaciones sexuales } \\
\hline Mensual & 0,0 & 30,6 & 35,7 & 31,8 \\
\hline 1 vez cada 15 días & 20,0 & 12,2 & 39,3 & 26,4 \\
\hline 1 vez por semana & 20,0 & 30,6 & 17,9 & 23,6 \\
\hline 2 veces por semana & 20,0 & 22,4 & 7,1 & 14,5 \\
\hline 3 veces por semana & 20,0 & 4,1 & 0,0 & 2,7 \\
\hline Diario & 20,0 & 0,0 & 0,0 & 0,9 \\
\hline \multicolumn{5}{|l|}{ Presencia de orgasmo } \\
\hline Depende de la posición & 60,0 & 44,9 & 57,1 & 51,8 \\
\hline Penetración profunda & 40,0 & 20,4 & 26,8 & 24,5 \\
\hline Nunca & 0,0 & 30,6 & 16,1 & 21,8 \\
\hline Al comienzo de la penetración & 0,0 & 4,1 & 0,0 & 1,8 \\
\hline Total & 100,0 & 100,0 & 100,0 & 100,0 \\
\hline
\end{tabular}

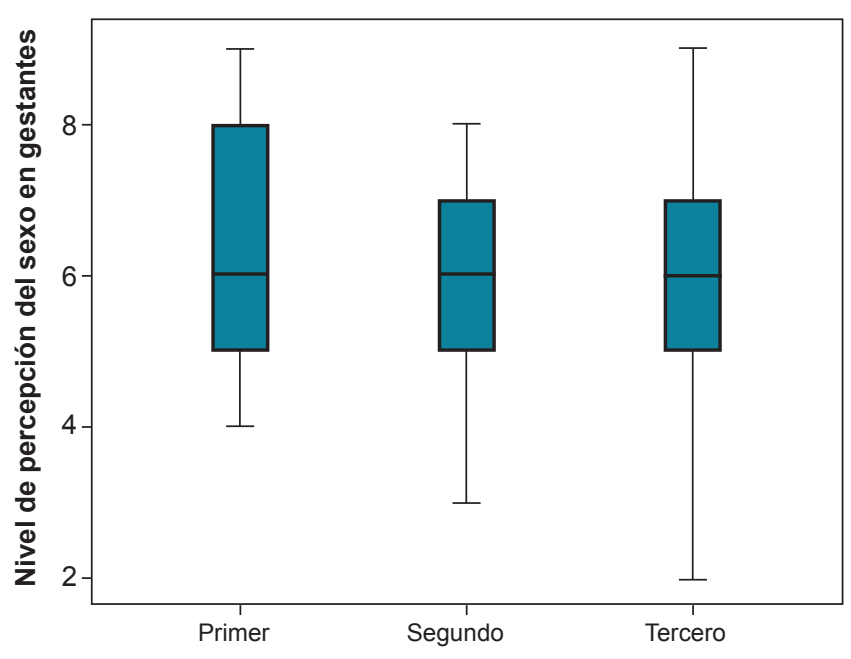

Trimestre de embarazo

\section{DISCUSIÓN}

Los efectos del embarazo sobre las funciones sexuales y sensaciones sexuales no son uniformes, para unos la gestación puede ser un periodo de mayor conciencia y goce sexual, en cambio otras no notan cambios o experimentan disminución de deseos sexuales. La gestación conlleva una serie de cambios anatómicos, funcionales y emocionales que pueden alterar su relación marital. Puede experimentar disconfort o dolor durante el coito y seguir consintiendo a su pareja, lo que podría condicionar desarmonía entre ellos ${ }^{4}$. Resulta importante indicar que más de la mitad consideran regular su vida sexual, es decir, que a pesar de las modificaciones y cambios que se producen como son el incremento del peso, los procesos cardiovasculares, psicológicos y bioquímicos, el proceso de la vida sexual se altera muy poco. Datos que difieren a lo encontrado por Valencia $^{6}$, donde el $96,6 \%$ de las 
gestantes que cursan el primer Trimestre, el $65.7 \%$ de las gestantes del segundo Trimestre y el $77,4 \%$ de las gestantes que cursan el tercer $\mathrm{T}$ tienen una actitud sexual negativa. En otro estudio realizado por Rodríguez el $47 \%$ de gestantes considera satisfactoria su vida sexual ${ }^{7}$.

Se tiene evidencia, que los cambios fisiológicos en el embarazo, afecta a la imagen personal de la gestante, pero este punto no se altera con la iniciativa para la actividad sexual, la cual hemos podido demostrar que se encuentra inalterada durante el embarazo. Uno de los eventos más estudiados durante el embarazo es la frecuencia de relaciones sexuales (RS), esta se reduce pero no en todas las gestantes. En el presente estudio la frecuencia de RS en el tercer trimestre, va disminuyendo de las que practican cada 15 días $(39,3 \%)$ a las que practican semanalmente $(17,9 \%)$, pero se incrementa en los que lo practican mensualmente $(35,7 \%)$. Probablemente los cambios fisiológicos del peso y aumento del abdomen hace que las parejas que tenían RS frecuentes por semana, vean reducida la misma y prefieran espaciarlo cada quince días o mensualmente. Datos muy diferentes encontrados por Uculmana ${ }^{8}$ donde refiere que el $36,4 \%$ tiene RS de 1 a 3 veces por semana. En el estudio realizado por Guivovich ${ }^{9}$ con una muestra de 1991 gestantes encontró que 1096 (70,6\%) gestantes del tercer trimestre tenían RS cada 11 o más días. Bello et $\mathrm{a}^{10}$ en su estudio sobre prácticas sexuales durante el embarazo en 375 gestantes Nigerianas encontraron que casi todas las gestantes tuvieron práctica sexual durante el embarazo $(91,6 \%)$.

Un evento que si sufre cambios significativos son los juegos previos antes de la actividad sexual, estos juegos son necesarios para el adecuado disfrute de la sexualidad, incluso durante el embarazo. Los juegos preliminares se reducen conforme se incrementan los trimestres de embarazo.

Uno de los eventos más frecuentemente estudiados durante el embarazo es la frecuencia de la actividad sexual, se puede indicar que esta se reduce, pero no en todas las gestantes. La frecuencia de la actividad sexual se reduce en las que lo practica más de una vez por semana, pero se incrementan en las que lo practicaban cada quince días o mensual. Probablemente los cambios fisiológicos del peso y adaptaciones fisiológicas, las parejas que tenía una actividad sexual frecuente por semana, vean reducida la misma, y prefieran espaciarlo cada quince días o mensualmente.

Se indican cambios significativos en lo referente a los juegos previos antes de la actividad sexual, estos juegos son necesarios para el adecuado disfrute de la sexualidad, incluso durante el embarazo. Los juegos preliminares se reducen conforme se incrementan los trimestres de embarazo. Así tenemos que en el primer trimestre el $100 \%$ indicó tener juegos preliminares en la mayoría de veces, en el segundo trimestre fue el 59,2\% y en el tercer trimestre llegó al 58,9\%, estadísticas muy similares encontradas en el estudio de Valencia ${ }^{6}$ donde manifiesta que el $69,0 \%$ de gestantes del primer trimestre prefieren como prácticas sexuales los besos y caricias y el $30,8 \%$ la manipulación de zonas excitables, el 40,0\% de las gestantes que cursan el segundo trimestre optan por los besos y caricias; al igual que las gestantes del tercer trimestre que prefieren los besos y caricias $(41,9 \%)$.

La presencia del orgasmo en la gestación en nuestro estudio depende mucho de la posición $(51,8 \%)$, la penetración profunda para obtención de un orgasmo llega a menos de la mitad con respecto a la anterior (24,5\%). Es importante resaltar que el $21,8 \%$ de gestantes nunca tuvieron orgasmo durante la gestación, resultados similares encontró Guivovich ${ }^{9}$ en su estudio donde el orgasmo y el placer sexual también disminuyen a medida que progresa el embarazo, incluso desde antes de la gestación $(6,1 \%)$ y el $(27,3 \%)$ de gestantes en el segundo trimestre refirieron no tener esta experiencia.

Es necesario incidir en la importancia de una adecuada educación sexual durante la atención preconcepcional (captando precozmente a la gestante) y en la atención psicoprofiláctica obstétrica una vez iniciado el embarazo, más aun sabiendo que la mayoría de gestantes se nutren de información sobre sexualidad a través de amigos y por el uso de internet y no por profesionales técnicamente capacitados. Por otra parte, una adecuada educación sexual de cada trimestre de embarazo puede ayudar a desmitificar los miedos maternos a causar lesiones fetales y a disfrutar de un embarazo y una sexualidad más plena y placentera.

Consideramos como posibles limitaciones del estudio el tamaño de la muestra, sobre todo para el primer trimestre, por lo que indicamos que para ese grupo los datos serían referenciales. Se concluye que la práctica sexual en el embarazo presenta modificaciones conforme se incrementan los trimestres del embarazo, siendo más marcados para los juegos preliminares y frecuencia sexual.

\section{AGRADECIMIENTOS.}

A la Licenciada Isabel Aparicio Ames de la Universidad Alas Peruanas de la filial Ica.

Financiamiento: Autofinanciado

Conflicto de interés: Los autores declaran no tener algún conflicto de intereses.

\section{REFERENCIAS BIBLIOGRÁFICAS}

1. Sacomori C, Luiz F. Prácticas sexuales de gestantes brasileñas. Rev Chil Obstet Ginecol 2008;73(5):313-317 
2. Rodrigues A, Knepper A, Luiz F. Perfil do comportamento sexual na gestacao. Fisioter Mov 2008;21(2):61-70

3. Sacomori C. Sexualidade na gestação: um olhar das ciências do movimento humano. (Tesis) Universidade do Estado de Santa Catarina. Brasil. 2009

4. Bello FA, Olayemi O, Aimakhu CO, Adekunle AO. Effect of pregnancy and childbird on sexuality of women in Ibadan, Nigeria. ISRN Obstet Gynecol 2011; 2011: 856-86

5. Gómez S. La expresión de la sexualidad durante la gestación y el puerperio. Cultura de cuidados. España 2012

6. Valencia A. Actitudes y prácticas sexuales según trimestre de embarazo en las gestantes que acuden al Centro de Salud Carlos Cueto Fernandini. 2015

7. Rodríguez M, Ramón E. Características y evolución del patrón sexual de la mujer embarazada. Revista electrónica trimestral de enfermería. $\mathrm{N}^{\circ} 32,2013$. España
8. Uculmana ZJ. Manifestaciones de sexualidad durante el IIIT de gestación en el Hospital María Auxiliadora. Abril mayo 2008

9. Guivovich A, Angulo T, Luján-Carpio E. Análisis de la actividad sexual de gestantes sin riesgo obstétrico que acuden a hospitales públicos de Lima Metropolitana, Perú. 2014

10. Bello F, Olayemi O, Aimakhu C, Adekunle A. Effect of pregnancy and childbirth on sexuality of women in Ibadan, Nigeria. Int Scholarly Research Network Obstet Gynecol 2011; article ID 856586; 1-6

\section{Correspondencia:}

Flor Alvarado Rodríguez. Dirección: Doña Catalina 426 Dpto. 302.

Santiago de Surco.

E-mail:fmar426@gmail.com 